\title{
SUPPLEMENT TEXT
}

One-sided test modification to avoid discordant effect sizes. Owen (2009) and Pearson (1934) applied a one-sided test form of Fisher's method to address the possible discordance issue. Two Fisher scores are first obtained from left and right one-sided pvalues: $S^{\text {Fisher } ; L}=-2 \sum_{k=1}^{K} \log \left(\tilde{p}_{k}\right)$ and $S^{\text {Fisher } ; R}=-2 \sum_{k=1}^{K} \log \left(1-\tilde{p}_{k}\right)$, where $\tilde{p}_{k}$ is the left-sided p-value of study $k$. The one-sided corrected Fisher score is defined as $S^{\text {Fisher; } C}=$ $\max \left(S^{\text {Fisher } ; L}, S^{\text {Fisher } ; R}\right)$. Below we similarly modify the rOP method for a one-sided corrected form. Denote by $S^{r O P ; L}=\tilde{p}_{(r)}$, where $\tilde{p}_{(r)}$ is the $r$ th order statistic of left one-sided p-values $\left\{\tilde{p}_{1}, \tilde{p}_{2}, \ldots, \tilde{p}_{K}\right\}$ from $K$ studies. Similarly, $S^{r O P ; R}=\tilde{q}_{(r)}$, where $\tilde{q}_{(r)}$ is the $r$ th order statistic of right one-sided p-values $\left\{\tilde{q}_{1}, \tilde{q}_{2}, \ldots, \tilde{q}_{K}\right\}=\left\{1-\tilde{p}_{1}, 1-\tilde{p}_{2}, \ldots, 1-\tilde{p}_{K}\right\}$ from $K$ studies. The test statistic is defined as $S^{r O P ; C}=\min \left(S^{r O P ; L}, S^{r O P ; R}\right)$. Under the null hypothesis that the one-sided p-values $\left\{\tilde{p}_{1}, \tilde{p}_{2}, \ldots, \tilde{p}_{K}\right\}$ are independently and uniformly distributed in $[0,1]$. The null distribution of $S^{r O P ; C}$ can be derived using integration by part. Equivalently, the null distribution could also be derived using the following property.

$$
\begin{aligned}
& \operatorname{Pr}\left(S^{r O P ; C} \leq p \mid H_{0}\right)=\operatorname{Pr}\left(S^{r O P ; L} \leq p \text { or } S^{r O P ; R} \leq p \mid H_{0}\right) \\
= & \operatorname{Pr}\left(\sum_{k=1}^{K} I\left(\tilde{p}_{k} \leq p\right) \geq r \text { or } \sum_{k=1}^{K} I\left(\tilde{p}_{k} \geq 1-p\right) \geq r \mid H_{0}\right)
\end{aligned}
$$

Because $\sum_{k=1}^{K} I\left(\tilde{p}_{k} \leq p\right) \geq r$ and $\sum_{k=1}^{K} I\left(\tilde{p}_{k} \geq 1-p\right) \geq r$ are not mutually exclusive (except when $r \geq[K / 2]+1$ and $p \leq 0.5$ ), the above probability should be calculated differently as follows.

1. For $r \geq[K / 2]+1$

(a) If $p \leq 0.5$, $\operatorname{Pr}\left(S^{r O P ; C} \leq p \mid H_{0}\right)=2 F(K-r ; K, 1-p)$, where $F(K-r ; K, 1-p)=\sum_{i=0}^{K-r}\left(\begin{array}{c}K \\ i\end{array}\right)(1-$ $p)^{i} p^{K-i}$ is the Binomial CDF for having $K-r$ successes in $K$ Bernoulli trails with success probability $1-p$.

(b) If $p>0.5$,

$\operatorname{Pr}\left(S^{r O P ; C} \leq p \mid H_{0}\right)=1-\sum_{i=K-r+1}^{r-1} \sum_{j=K-r+1}^{K-i} \frac{K !}{i ! j !(K-i-j) !}(1-p)^{i+j}(2 p-1)^{K-i-j}$.

2. For $r \leq[K / 2]$

(a) If $p \leq 0.5$, $\operatorname{Pr}\left(\bar{S}^{r O P ; C} \leq p \mid H_{0}\right)=1-\sum_{i=0}^{r-1} \sum_{j=0}^{r-1} \frac{K !}{i ! j !(K-i-j) !} p^{i+j}(1-2 p)^{K-i-j}$.

(b) If $p>0.5$, $\operatorname{Pr}\left(S^{r O P ; C} \leq p \mid H_{0}\right)=1$. 


\section{References.}

Owen, A. B. (2009). Karl Pearson's meta-analysis revisited. The Annals of Statistics 37 3867-3892.

Pearson, K. (1934). ON A NEW METHOD OF DETERMINING "GOODNESS OF FIT.". Biometrika 26425. 